\title{
Assessment of rumen bacteria in dairy cows with varied milk protein yield
}

\author{
M. Y. Xue,${ }^{1}$ H. Z. Sun, ${ }^{2}$ X. H. Wu, ${ }^{1}$ L. L. Guan, ${ }^{2 *}$ and J. X. Liu ${ }^{1 *}$ \\ ${ }^{1}$ Institute of Dairy Science, MoE Key Laboratory of Molecular Animal Nutrition, College of Animal Sciences, Zhejiang University, Hangzhou, \\ 310058, China \\ ${ }^{2}$ Department of Agricultural, Food and Nutritional Science, University of Alberta, Edmonton, AB T6G 2P5, Canada
}

\section{ABSTRACT}

The present study was conducted to assess rumen bacteria in lactating cows with different milk protein yield, aiming to understand the role of rumen bacteria in this trait. Cows with high milk protein yield (high milk yield and high milk protein content, $\mathrm{HH} ; \mathrm{n}=20$ ) and low milk protein yield (low milk yield and low milk protein content, LL; $\mathrm{n}=20$ ) were selected from 374 mid-lactation Holstein dairy cows fed a high-grain diet. Measurement of the rumen fermentation products showed that the concentrations of ruminal total volatile fatty acids, propionate, butyrate, and valerate and the proportion of isobutyrate were higher in the $\mathrm{HH}$ cows than in the LL cows. Amplicon sequencing analysis of the rumen bacterial community revealed that the richness (Chao 1 index) of rumen microbiota was higher in the LL cows than in the HH cows. Among the 10 predominant bacterial phyla (relative abundance being $>0.10 \%$, present in $>60 \%$ of animals within each group), the relative abundance of Proteobacteria was 1.36-fold higher in the HH cows than in the LL cows. At the genus level, the relative abundance of Succinivibrio was significantly higher and that of Clostridium tended to be higher in the LL cows than in the $\mathrm{HH}$ cows. Sharpea was 2.28-fold enriched in the HH cows compared with the LL cows. Different relationships between the relative abundances of rumen microbial taxa and volatile fatty acid concentrations were observed in the HH and the LL animals, respectively. Succinivibrio and Prevotella were positively correlated with acetate, propionate, and valerate in the LL cows, whereas Sharpea was positively correlated with propionate and valerate concentrations in the $\mathrm{HH}$ cows. Collectively, our results revealed that rumen bacterial richness and the relative abundances of several bacterial taxa significantly differed between dairy cows with high and low milk protein yields, suggesting the potential roles

Received November 11, 2018.

Accepted February 20, 2019.

*Corresponding authors: lguan@ualberta.ca and liujx@zju.edu.cn of rumen microbiota contributing to milk protein yield in dairy cows.

Key words: $16 \mathrm{~S}$ rRNA gene sequencing, rumen bacteria, dairy cow, milk protein yield

\section{INTRODUCTION}

Milk protein is considered to be an important milk component and a key economic trait. Many efforts have been made to increase milk protein yield (MPY; milk protein content $\times$ milk yield), with the advancements being made to enhance either milk yield or milk protein via improvements in genetics (Gernand and Konig, 2014), herd management (Gillah et al., 2014), and nutrition (Sova et al., 2013). Although milk protein and milk yield are negatively correlated in dairy production (Bedö et al., 1995), Wu et al. (2018) showed that there were cows with high MPY at both high milk protein content and high milk yield levels (defined as high MPY in this study) within a herd when the cows were fed the same diet. From the same study, Wu et al. (2018) reported several serum biomarkers (metabolites) related to high MPY and revealed the potential metabolic pathways affecting milk protein synthesis, suggesting the possibility of selecting cows with high MPY.

It has been reported that ruminal VFA and microbial proteins derived from microbial fermentation are key factors that directly affect milk biosynthesis (Flint and Bayer, 2008). Microorganisms inhabiting the rumen, including a diverse range of bacteria, archaea, protozoa, and fungi, derive nutrients from indigestible plant feedstuffs for dairy cows (Russell and Rychlik, 2001). Within the rumen microbiome, bacteria are the major contributor to producing VFA and microbial proteins (Kim et al., 2011). Our recent study found the core and noncore rumen bacteriome from a large population of mid-lactation cows that were fed the same high-grain diet under the same management (Xue et al., 2018), suggesting that rumen fermentation is varied when cows are fed the same diet, which could lead to varied milking traits. Although the linkages between rumen 
microbiota and milking traits of dairy cows have revealed that specific bacterial taxa are associated with milk yield and milk composition (Jami et al., 2014; Jewell et al., 2015), it is unknown whether the rumen microbiota also plays a role in MPY.

We hypothesized that rumen microbiota differed in cows with high MPY and low MPY (low milk protein content and low milk yield) and that the varied microbiota could affect their microbial fermentation metabolites (VFA) that contribute to milk protein and milk yield as well as MPY. The objective of the current study was to compare the rumen bacterial composition between cows with high and low MPY using 16S rRNA gene sequencing and to assess whether the difference may lead to varied VFA production.

\section{MATERIALS AND METHODS}

\section{Animals and Lactation Data}

The animal care and experimental procedures were approved by the Animal Care Committee of Zhejiang University (Hangzhou, China) and were in accordance with the university's guidelines for animal research. A total of 374 healthy mid-lactation Holstein dairy cows $($ parity $=3.08 \pm 1.14, \mathrm{DIM}=159 \pm 34$, mean $\pm \mathrm{SD})$ housed at a commercial dairy farm were used. The feeding and management of the experimental cows have been described previously (Wu et al., 2018). Briefly, the animals were fed the same diet with a concentrate-toforage ratio of 57:43 (DM basis) and were under the same management regimen. Milk yields were recorded for 3 consecutive days, and milk samples were collected on the third day at a volume ratio of 4:3:3 corresponding to the morning $(0630 \mathrm{~h})$, afternoon $(1400 \mathrm{~h})$, and evening $(2000 \mathrm{~h})$ milkings. Milk components (milk protein, milk fat, and lactose) were measured using infrared analysis with a spectrophotometer (Foss-4000; Foss Electric A/S, Hillerød, Denmark), and MPY was calculated using the milk protein content multiplied by milk yield measured only for the third day. Based on milk yield and milk protein content, 20 high-MPY (cows with high milk yield and high milk protein content; HH) cows (parity $=2.6 \pm 0.26, \mathrm{DIM}=160 \pm$ 6 ) and 20 low MPY (cows with low milk yield and low milk protein content; LL) cows (parity $=3.9 \pm 0.35$, $\mathrm{DIM}=160 \pm 7$ ) were selected for further analysis. The statistical power of 20 animals within each group was calculated as reported previously (Wu et al., 2018), with a power of $99 \%$ for milk yield and $99 \%$ for milk protein. Milk yield and milk protein content of the $\mathrm{HH}$ and LL cows were reported previously (Wu et al., 2018), with milk yield being $>34.5 \mathrm{~kg} / \mathrm{d}$ for the $\mathrm{HH}$ cows and $<31$ $\mathrm{kg} / \mathrm{d}$ for the LL cows and milk protein content being
$>3.20 \%$ for the HH cows and $<2.90 \%$ for the LL cows (Supplemental Table S1 and Supplemental Figure S1, https://doi.org/10.3168/jds.2018-15974).

\section{Sampling of Rumen Contents and Measurement of Fermentation Variables}

The rumen content was collected before the morning feeding on the third day of the 3 consecutive days using an oral stomach tube (Shen et al., 2012). The rumen content samples were then stored at $-80^{\circ} \mathrm{C}$ until further analysis. The ammonia $\mathrm{N}$ concentrations of the 40 animals were determined using steam distillation into boric acid and titration with dilute hydrochloric acid, and the VFA concentrations of the 40 animals were measured using GC (GC-2010, Shimadzu, Kyoto, Japan; Hu et al., 2005).

\section{DNA Extraction and 16S rRNA Gene Sequencing}

Total DNA was extracted using a repeated beadbeating plus column method (Yu and Morrison, 2004). This method uses cell lysis by bead beating in the presence of high concentrations of SDS, salt, and EDTA. The subsequent DNA was purified using a QIAamp DNA Stool Mini Kit (Qiagen, Hilden, Germany). The qualities and quantities of the DNA samples were measured using the NanoDrop 2000 spectrophotometer (NanoDrop Technologies, Wilmington, DE). The amplicon DNA was amplified using the $341 \mathrm{~F} / 806 \mathrm{R}$ primer set (5'-CCTAYGGGRBGCASCAG-3' $/ 5^{\prime}$ GGACTACNNGGGTATCTAAT-3'), with the reverse primer containing a 6-bp error-correcting barcode unique to each sample. The PCR reaction was performed using Phusion High-Fidelity PCR Mastermix [New England Biolabs (Beijing) Ltd., China] under the following cycling conditions: $94^{\circ} \mathrm{C}$ for $3 \mathrm{~min}$; 35 cycles at $94^{\circ} \mathrm{C}$ for $45 \mathrm{~s}, 50^{\circ} \mathrm{C}$ for $60 \mathrm{~s}$, and $72^{\circ} \mathrm{C}$ for $90 \mathrm{~s}$; and $72^{\circ} \mathrm{C}$ for $10 \mathrm{~min}$. The PCR products were visualized on $2 \%$ agarose gels and purified using a QIAquick gel extraction kit (Qiagen). Amplicon sequencing was conducted on an Illumina (San Diego, CA) HiSeq platform using the pair-ended $2 \times 250$ bp at Novogene Bioinformatics Technology Co. Ltd. (Beijing, China). The raw sequences were deposited in the NCBI Sequence Read Archive (https://www.ncbi.nlm.nih.gov/sra/ SRP149811) under the accession number SRP149811.

\section{Processing of Sequencing Data}

Pair-end reads were merged by FLASH (Magoč and Salzberg, 2011). Sequences were demultiplexed and quality filtered using QIIME (http://qiime.org), and bases with quality scores higher than 20 were retained 
for further analysis (Caporaso et al., 2010). Chimeric sequences were identified and removed using UCHIME (Edgar et al., 2011). Operational taxonomic units (OTU) were clustered with a $97 \%$ similarity threshold using UPARSE (Edgar, 2013), and taxonomy was assigned using the latest Greengenes database (May 2013 release; http://greengenes.lbl.gov).

The alpha diversity of the bacterial communities was obtained using various diversity indices (Chao 1, abundance-based coverage estimator, Shannon, and observed species). To generally compare the bacterial communities between the 2 groups, the jackknifed beta diversity was performed based on Bray-Curtis dissimilarity and visualized by principal coordinate analysis using QIIME. The composition of bacterial phyla was visualized using Circos (version 0.69-6; Krzywinski et al., 2009). To detect the relationship of VFA and milking traits with bacterial communities, redundancy analysis (RDA) was performed at genus level using the R program (https://www.r-project.org) vegan package, with VFA proportions, milk yield, milk protein content and MPY considered as explanatory variables. The correlation heatmaps were generated using the $\mathrm{R}$ program pheatmap package.

\section{Statistical Analysis}

Milk yield, milk composition, ammonia N, and VFA concentrations and individual proportions between the $\mathrm{HH}$ and LL cows were analyzed using $t$-test, with $P<$ 0.05 defined as statistical significance. The alpha diversity indices of bacterial communities were compared between the 2 groups using Wilcoxon rank-sum test, with
$P<0.05$ defined as statistical significance. Analysis of similarity was performed based on genus-level bacterial taxa using the $\mathrm{R}$ program vegan package. Bacterial genera and OTU with relative abundances greater than $0.10 \%$ in at least $60 \%$ of the cows within each group were used for further comparative analysis. The relative abundances of bacterial taxa were compared using the Wilcoxon rank-sum test, and $P$-values were corrected for a false discovery rate using the Benjamini-Hochberg method, with false discovery rate-corrected $P<0.05$ being considered significant. Correlation analysis among the selected bacterial taxa, VFA concentrations, and milking traits was performed using Spearman's rank correlation.

\section{RESULTS}

\section{Rumen Fermentation Variables}

Table 1 presents the rumen fermentation variables of dairy cows with different MPY. The concentrations of total VFA $(P=0.03)$, propionate $(P=0.02)$, butyrate $(P=0.04)$, and valerate $(P=0.02)$ as well as the proportion of isobutyrate $(P=0.01)$ were higher in the rumen of the HH cows than in the rumen of the LL cows (Table 1 ). We did not find a difference in ruminal ammonia $\mathrm{N}(P=0.92)$ between 2 groups.

\section{Rumen Bacterial Compositions}

Amplicon sequencing of the partial 16S rRNA gene generated a total of 2,417,180 high-quality sequences and an average of $71,933 \pm 1,987$ sequences per sample,

Table 1. Comparison of rumen fermentation variables in HH and LL dairy cows

\begin{tabular}{lcccc}
\hline & \multicolumn{2}{c}{ Group $^{1}$} & & \\
\cline { 2 - 3 } Item & HH & LL & SEM & P-value \\
\hline Ammonia N, mg/dL & 6.82 & 6.78 & 0.605 & 0.922 \\
Concentration, mmol/L & & & & \\
$\quad$ Total VFA & 108.7 & 94.7 & 8.260 & 0.029 \\
Acetate & 74.3 & 66.0 & 5.447 & 0.050 \\
Propionate & 19.2 & 15.6 & 2.092 & 0.019 \\
Butyrate & 11.5 & 9.7 & 1.129 & 0.036 \\
Isobutyrate & 0.89 & 0.92 & 0.067 & 0.640 \\
Valerate & 1.24 & 1.06 & 0.104 & 0.017 \\
Isovalerate & 1.50 & 1.36 & 0.114 & 0.106 \\
Molar proportion, \% & & & & \\
Acetate & 68.5 & 69.9 & 1.144 & 0.095 \\
Propionate & 17.6 & 16.3 & 1.148 & 0.133 \\
Butyrate & 10.6 & 10.1 & 0.543 & 0.263 \\
Isobutyrate & 0.83 & 1.02 & 0.089 & 0.007 \\
Valerate & 1.141 & 1.138 & 0.073 & 0.955 \\
Isovalerate & 1.38 & 1.50 & 0.098 & 0.113 \\
Acetate:propionate & 4.02 & 4.38 & 0.334 & 0.137 \\
\hline I & & & & \\
\end{tabular}

${ }^{1} \mathrm{HH}=$ dairy cows with high milk yield and high protein yield; LL = dairy cows with low milk yield and low protein yield. 
Table 2. Valid operational taxonomic units (OTU) and alpha diversity indices of rumen bacteria in $\mathrm{HH}$ and LL dairy cows

\begin{tabular}{|c|c|c|c|c|}
\hline \multirow[b]{2}{*}{ Item } & \multicolumn{2}{|c|}{ Group $^{1}$} & \multirow[b]{2}{*}{ SEM } & \multirow[b]{2}{*}{$P$-value } \\
\hline & $\mathrm{HH}$ & LL & & \\
\hline OTU & 1,554 & 1,618 & 14.94 & 0.029 \\
\hline Chao1 & 1,817 & 1,904 & 20.12 & 0.035 \\
\hline Shannon & 8.56 & 8.77 & 0.074 & 0.157 \\
\hline
\end{tabular}

${ }^{1} \mathrm{HH}=$ dairy cows with high milk yield and high protein yield; LL = dairy cows with low milk yield and low protein yield.

which were assigned to 2,855 OTU. The number of OTU was significantly higher in the LL cows than in the HH cows $(P=0.03$; Table 2$)$. The number of $16 \mathrm{~S}$ rRNA gene sequences was sufficient to uncover more than $98.2 \%$ of bacterial taxa, with Good's coverage values ranging from 98.2 to $99.3 \%$.

Taxonomic analysis identified that the sequences belonged to 15 bacterial phyla and 49 bacterial genera, accounting for $99.72 \pm 0.13$ and $46.6 \pm 5.75 \%$ of the total bacterial sequences, respectively (Supplemental Table S2, https://doi.org/10.3168/jds.2018-15974). The predominant bacterial phyla consisted of 10 taxa (with a relative abundance being $>0.10 \%$ in more than $60 \%$ of the animals within each group), with Firmicutes $(49.10 \%), \quad$ Bacteroidetes (34.89\%), Proteobacteria (9.32\%), Tenericutes $(2.01 \%)$, and Spirochaetes $(1.29 \%)$ being the most abundant (Supplemental Table S2, https: //doi.org/10.3168/jds.2018-15974). The predominant bacterial taxa at genus level (with a relative abundance being $>0.10 \%$ in more than $60 \%$ of the animals within each group) consisted of 29 genera, with Prevotella (20.12\%), Ruminococcus (8.15\%), Butyrivibrio (3.18\%), Coprococcus (2.06\%), Shuttleworthia (1.28\%), and Clostridium $(1.32 \%)$ being the most abundant (Table 3).

\section{Comparison of the Rumen Microbiota Between Cows with Different MPY}

The microbial diversity indices were first compared. The principal coordinate analysis did not show clear separations of rumen bacteriome between the $\mathrm{HH}$ and LL cows at genus level based on the Bray-Curtis dissimilarity matrices (Figure 1A). Furthermore, analysis of similarity (based on Bray-Curtis dissimilarity) showed no significant difference in microbial profiles between the 2 groups $(\mathrm{R}=0.024, P=0.197$; Figure 1B). However, the richness index (Chao 1) was higher in the LL cows than in the HH cows $(P=0.03$; Table 2).

When the relative abundances of bacterial phyla were compared using the Wilcoxon rank-sum test, no significant difference was found between the $\mathrm{HH}$ and
LL cows (Figure 2A). At genus level, the relative abundance of Succinivibrio $(0.22 \%$ in HH cows vs. $0.29 \%$ in LL cows; corrected $P=0.019)$ was higher and that of Clostridium (1.23\% in HH cows vs. $1.42 \%$ in LL cows; corrected $P=0.062$ ) tended to be higher in the LL cows than in the HH cows (Figure 2B; Table 3). In addition, the relative abundances of 13 OTU were different between the HH and LL cows (corrected $P<$ 0.05; Supplemental Table S3, https://doi.org/10.3168/ jds.2018-15974), among which 6 were classified at genus level and the other 7 could be identified only at family, order, and phylum levels.

\section{Relationships of MPY with Rumen Bacterial Community and Metabolites}

Because the higher number of OTU was not classified, the bacterial taxa that were classified at genus level were subjected to the following correlation analysis. The correlations among rumen bacterial taxa (those with relative abundances $>0.10 \%$, present in $>60 \%$ of animals in each group), VFA, and milking traits were evaluated by RDA. Among the VFA, propionate $($ contribution $=71.4 \% ; P=0.001)$ and valerate (contribution $=68.5 \% ; P=0.001$ ) contributed to the compositional differences in rumen bacteriome at genus level between 2 groups (Figure 3 ). Milk yield, milk protein (Figure 3A), and MPY (Figure 3B) did not contribute to the rumen bacterial community compositional differences. Unclassified genera within the family Succinivibrionaceae (contribution $=65.7 \% ; P=0.001$ ), along with the genus Prevotella (contribution $=37.2 \%$; $P=0.04)$, were the main contributors to the bacterial compositional differences in the rumen between 2 groups (Figure 3).

Spearman correlation analysis revealed various significant correlations between the relative abundances of the observed differential bacterial genera and VFA concentrations and milking traits within each group (Figure 4). In the $\mathrm{HH}$ cows, the relative abundances of Sharpea and unclassified Succinivibrionaceae were positively correlated with propionate and valerate $(0.52$ $<\mathrm{R}<0.78 ; P<0.01$ ), and the relative abundance of Succinivibrio was positively correlated with butyrate (R $=0.41 ; P<0.006$; Figure 4A). In the LL cows, the relative abundance of Prevotella was positively correlated with acetate, propionate, and valerate $(0.51<\mathrm{R}<$ 0.71; $P<0.05)$; the relative abundance of Succinivibrio was positively correlated with milk protein, butyrate, and valerate $(0.49<\mathrm{R}<0.68, P<0.05)$; and the relative abundance of unclassified Succinivibrionaceae was positively correlated with propionate and valerate $(0.53<\mathrm{R}<0.65 ; P<0.05)$. 


\section{DISCUSSION}

The current study characterized the rumen bacterial composition in cows with different MPY. It is noticeable that the oral stomach tube was used to collect the rumen content samples (Shen et al., 2012), although cannulation is considered to be a preferred method for collecting representative samples of rumen contents (Geishauser and Gitzel, 1996). Studies have compared the fermentation and microbiota between the 2 widely used methods and showed either different (Geishauser and Gitzel, 1996; Duffield et al., 2004) or similar (LodgeIvey et al., 2009; Terré et al., 2013; Ramos-Morales et al., 2014) results. Nevertheless, stomach tubing is a less invasive method than rumen cannulation (Shen et al., 2012) when sampling a large population. Additionally, due to the potential undersampling of rumen contents (1 sample/cow) in the current study, future studies based on sufficient samplings (different sampling loca- tions and sampling time) are needed to provide more representative profiles of the rumen bacterial community.

Further, we identified varied bacterial diversity and specific rumen bacteria that may contribute to MPY. Although the parity was significantly different between the 2 groups $(\mathrm{HH}=2.60 \pm 0.26, \mathrm{LL}=3.90 \pm 0.35 ; P$ $=0.004$ ), our previous study showed that parity does not affect rumen bacteriome composition (Xue et al., 2018), indicating that the difference in parity should not be a factor affecting the identified bacterial compositional differences between HH and LL cows. First, comparison of the alpha diversity indices revealed a lower richness (Chao 1 index) in cows with high MPY. A lower richness of the rumen microbiota has been observed in dairy cows with higher VFA production (Shabat et al., 2016), higher milk yield, and lower milk fat (Xue et al., 2018). The low richness of microbiota in the rumen has also been reported in cattle with higher

Table 3. Comparison of rumen bacterial genera ${ }^{1}$ between HH and LL dairy cows

\begin{tabular}{|c|c|c|c|c|}
\hline \multirow[b]{2}{*}{ Bacterial taxa } & \multicolumn{2}{|c|}{ Group, ${ }^{2} \%$} & \multirow[b]{2}{*}{ SEM } & \multirow[b]{2}{*}{ Adjusted $P$-value ${ }^{3}$} \\
\hline & HH & LL & & \\
\hline \multicolumn{5}{|l|}{ Firmicutes } \\
\hline Ruminococcus & 8.29 & 8.02 & 0.270 & 0.607 \\
\hline Butyrivibrio & 3.15 & 3.20 & 0.181 & 0.850 \\
\hline Coprococcus & 1.96 & 2.16 & 0.082 & 0.449 \\
\hline Clostridium & 1.23 & 1.42 & 0.049 & 0.062 \\
\hline Shuttleworthia & 1.28 & 1.29 & 0.131 & 0.570 \\
\hline Lachnospira & 0.81 & 0.84 & 0.078 & 0.871 \\
\hline Pseudobutyrivibrio & 0.83 & 0.78 & 0.032 & 0.745 \\
\hline Succiniclasticum & 0.64 & 0.83 & 0.061 & 0.130 \\
\hline Oscillospira & 0.58 & 0.65 & 0.025 & 0.194 \\
\hline Anaerostipes & 0.41 & 0.42 & 0.027 & 0.358 \\
\hline RFN20 & 0.37 & 0.36 & 0.020 & 0.829 \\
\hline Moryella & 0.35 & 0.34 & 0.015 & 0.766 \\
\hline Bulleidia & 0.20 & 0.20 & 0.010 & 0.705 \\
\hline Selenomonas & 0.19 & 0.20 & 0.011 & 0.279 \\
\hline p-75-a5 & 0.18 & 0.18 & 0.010 & 0.787 \\
\hline L7A_E11 & 0.15 & 0.15 & 0.008 & 0.957 \\
\hline Anaerovibrio & 0.13 & 0.14 & 0.006 & 0.304 \\
\hline Sharpea & 0.16 & 0.07 & 0.035 & 0.957 \\
\hline Unclassified Lachnospiraceae & 0.10 & 0.10 & 0.030 & 0.808 \\
\hline \multicolumn{5}{|l|}{ Bacteroidetes } \\
\hline Prevotella & 21.00 & 19.25 & 1.178 & 0.589 \\
\hline CF231 & 0.98 & 1.14 & 0.072 & 0.465 \\
\hline YRC22 & 0.80 & 0.72 & 0.042 & 0.372 \\
\hline \multicolumn{5}{|l|}{ Proteobacteria } \\
\hline Succinivibrio & 0.22 & 0.29 & 0.017 & 0.019 \\
\hline Ruminobacter & 0.16 & 0.14 & 0.010 & 0.402 \\
\hline \multicolumn{5}{|l|}{ Fibrobacteres } \\
\hline Fibrobacter & 0.84 & 0.94 & 0.064 & 0.829 \\
\hline \multicolumn{5}{|l|}{ Tenericutes } \\
\hline Anaeroplasma & 0.44 & 0.45 & 0.024 & 0.330 \\
\hline
\end{tabular}

${ }^{1}$ Bacterial genera with relative abundance greater than $0.10 \%$ in at least $60 \%$ of animals within each group were regarded as detected and used for the comparison.

${ }^{2} \mathrm{HH}=$ dairy cows with high milk yield and high protein yield; LL = dairy cows with low milk yield and low protein yield.

${ }^{3}$ Adjusted $P$-value $=$ false discovery rate-adjusted $P$-value. The $P$-values between $\mathrm{HH}$ and LL cows were obtained using Wilcoxon rank-sum test. 

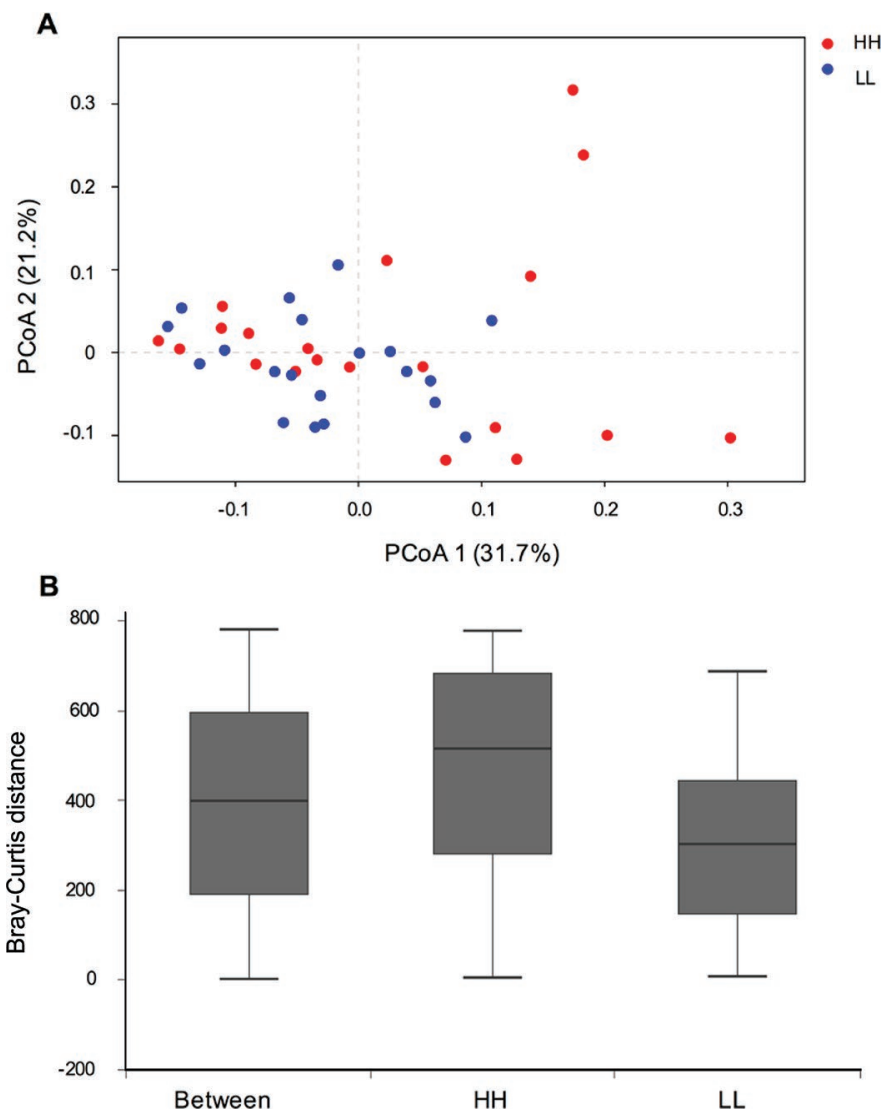

Figure 1. Comparison of the composition of bacterial genera in the rumen of dairy cows. (A) Principal coordinate analysis (PCoA) of bacterial genera. (B) Analysis of similarity of bacterial genera between the 2 groups of cows ( $\mathrm{HH}=$ dairy cows with high milk yield and high protein yield; $\mathrm{LL}=$ dairy cows with low milk yield and low protein yield; $\mathrm{R}=0.024 ; P=0.197)$. Boxes represent the interquartile range between the first quartile and the third quartile. The bands inside the boxes define the median. Whiskers represent the minimum and maximum of Bray-Curtis distance.

feed efficiency (Shabat et al., 2016; Li and Guan, 2017), suggesting that the HH cows may have higher feed efficiency than the LL cows. Indeed, higher concentrations of VFA (total VFA, propionate, butyrate, and valerate) were found in the $\mathrm{HH}$ cows than in the LL cows, further supporting that these cows may have higher ruminal fermentation efficiency. However, it is noticeable that the ruminal VFA concentrations are the result of microbial production and host absorption. Future studies to examine VFA absorption are needed to determine the relationship between microbial VFA production and host utilization and their roles in high MPY.

Although the analysis of similarity indicated that interindividual variations existed within each group, differences in rumen bacterial taxa between 2 groups were identified. Here, we focused on genus level rather than OTU level because a large number of OTU $(52.84 \%$ of sequences) were not classified at either genus or deeper taxonomic levels. The identified differences in the abundances of some rumen bacterial taxa suggested that specific bacteria might be associated with MPY. For example, 1.36-fold enrichment of the Proteobacteria phylum $(7.88 \%$ in the LL cows vs. $10.75 \%$ in the $\mathrm{HH}$ cows) was found in the rumen of the $\mathrm{HH}$ cows, with the family Succinivibrionaceae being the most abundant within this phylum $(7.13 \%$ in the LL cows vs. $9.95 \%$ in the HH cows). Succinivibrionaceae ferments carbohydrates to produce succinate (a precursor of propionate) and acetate, and its higher abundance has been linked to lower methane emission, accompanied by changes in acetate and hydrogen production (Wallace et al., 2015). Genera belonging to the Succinivibrionaceae family mainly include 4 groups (Succinivibrio, Ruminobacter, Anaerobiospirillum, and Succinimonas), among which Succinivibrio and Ruminobacter were identified in the present study, with low relative abundances $(0.26$ and $0.15 \%$, respectively). From these 2 genera, the relative abundance of Succinivibrio was positively associated with milk protein in this study. Population of Succinivibrio sp. in the rumen has been reported to be positively associated with feed efficiency of beef cattle (Hernandez-Sanabria et al., 2012) and dairy cows (Elolimy et al., 2018) due to its function of producing succinate, the precursor of propionate. Propionate is the main precursor for gluconeogenesis in the liver, which is vital for cow milk production. Based on higher rumen propionate concentration in the $\mathrm{HH}$ cows than in the LL cows, the relative abundance of Succinivibrio was speculated to be higher in the HH cows than in the LL cows. However, the higher abundances of Succinivibrio and 1 OTU belonging to this genus were found in the LL cows, which was not consistent with our speculation. Such discrepancy may be due to the fact that the taxonomic assessment was based only on genus level. The Succinivibrio genus has several species and strains, among which Succinivibrio dextrinosolvens is the most well studied that produces succinate (Bryant and Small, 1956), whereas the functions of other unclassified species belonging to this genus remain unknown (Nyonyo et al., 2013). Because different species may have different functions, identification at deeper taxonomic levels is required to detect whether and how the Succinivibrio species could be attributed to MPY. In addition to the above-classified genera, most members belonging to the Succinivibrionaceae family were unclassified (average relative abundance of $7.87 \%$ ). In our previous study, we found that unclassified Succinivibrionaceae was positively associated with propionate in 334 lactating dairy cows (Xue et al., 2018). The associations found herein between Succinivibrionaceae, VFA, and MPY were consistent with this previous study. Our results indicated the role of this taxon in propionate produc- 


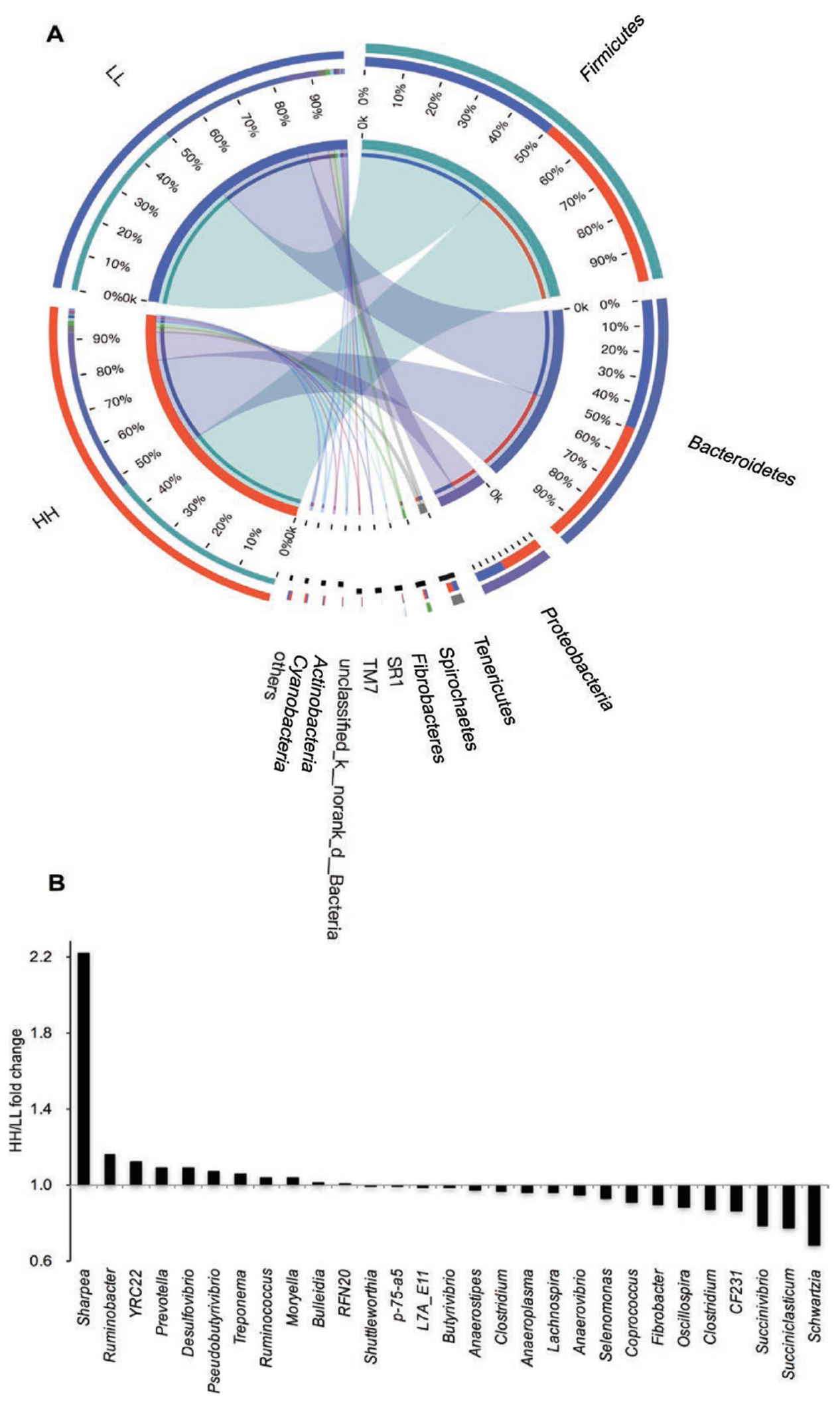

Figure 2. Comparison of the abundances of rumen bacterial phyla and genera between the 2 groups. (A) Bacterial phyla (relative abundance $>0.1 \%$ in at least $60 \%$ of the animals within each group) of 2 the groups visualized using Circos (Krzywinski et al., 2009). (B) Fold change (HH/ LL) in the relative abundances of the bacterial genera (relative abundance $>0.10 \%$ in at least $60 \%$ of the animals within each group). $\mathrm{HH}=$ dairy cows with high milk yield and high protein yield; LL = dairy cows with low milk yield and low protein yield. 
tion, and the propionate production might influence the gluconeogenesis in the liver and the glucose supplied for lactogenesis, which could affect MPY.

The RDA and Spearman correlation analysis indicated that Prevotella might positively affect VFA concentrations (acetate, propionate, and valerate). Prevotella is a genus consisting of proteolytic, amylolytic, and hemicellulolytic bacteria dominating the rumen of adult dairy cows and producing succinate and acetate (Stevenson and Weimer, 2007). In dairy cows, the abundances of 14 and 11 OTU belonging to Prevotella have been reported to be strongly associated with high gross feed efficiency (ECM/DMI) and low gross feed efficiency, respectively (Jewell et al., 2015). The abundance of Prevotella also has been reported to be negatively associated with milk yield (Jami et

A
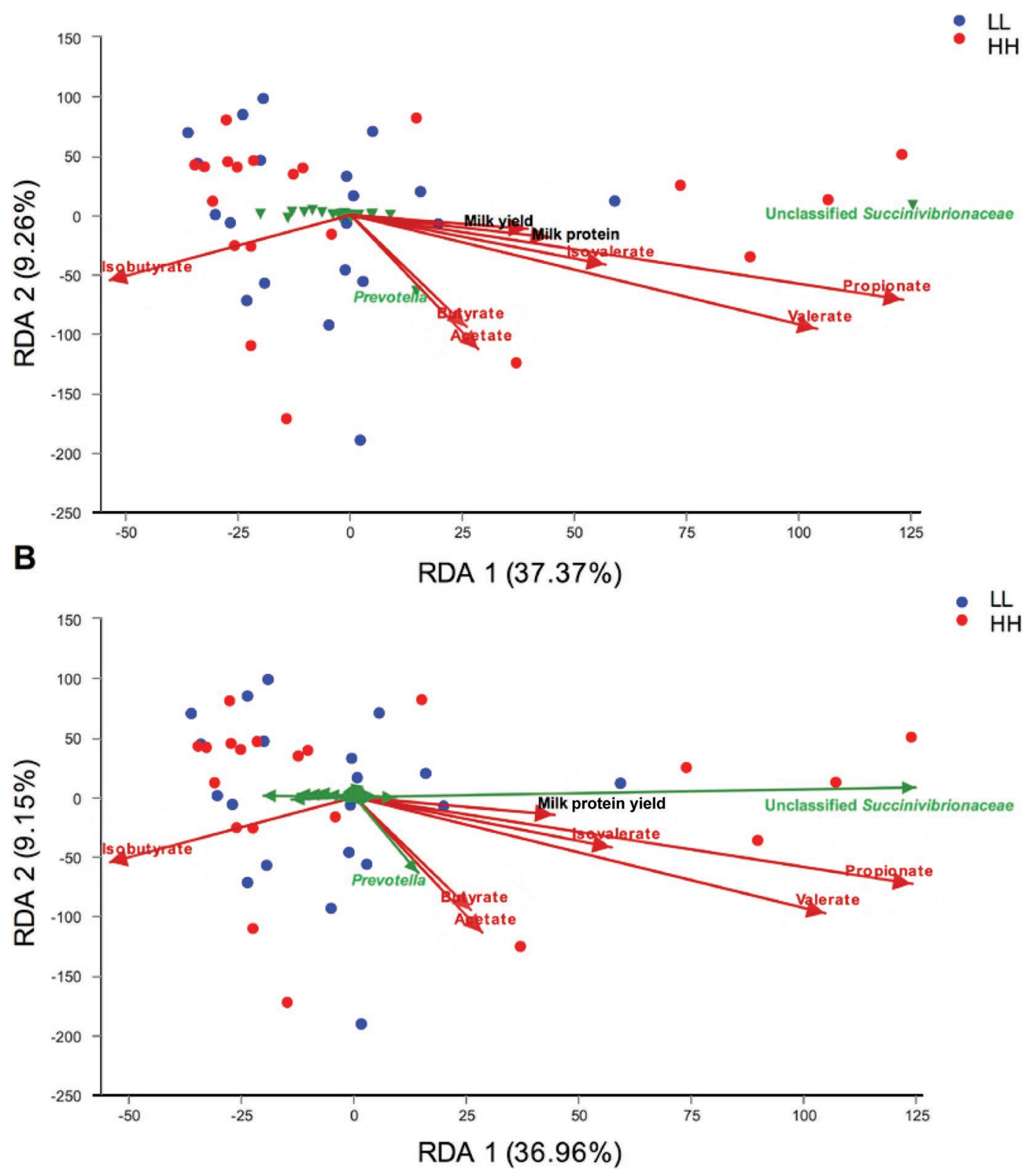

Figure 3. Relationship between rumen bacterial taxa, VFA concentrations, and milking traits. (A) Biplot of the redundancy analysis (RDA) showed the relationships between bacterial genera, VFA concentrations, and milk yield and milk protein content. (B) Biplot of the RDA showed the relationships between bacterial genera, VFA concentrations, and milk protein yield. $\mathrm{HH}=$ dairy cows with high milk yield and high protein yield; LL = dairy cows with low milk yield and low protein yield. 
A

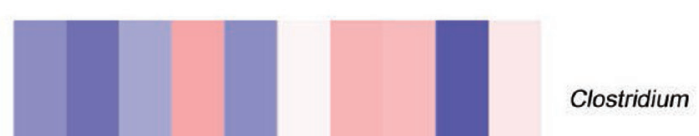

Clostridium
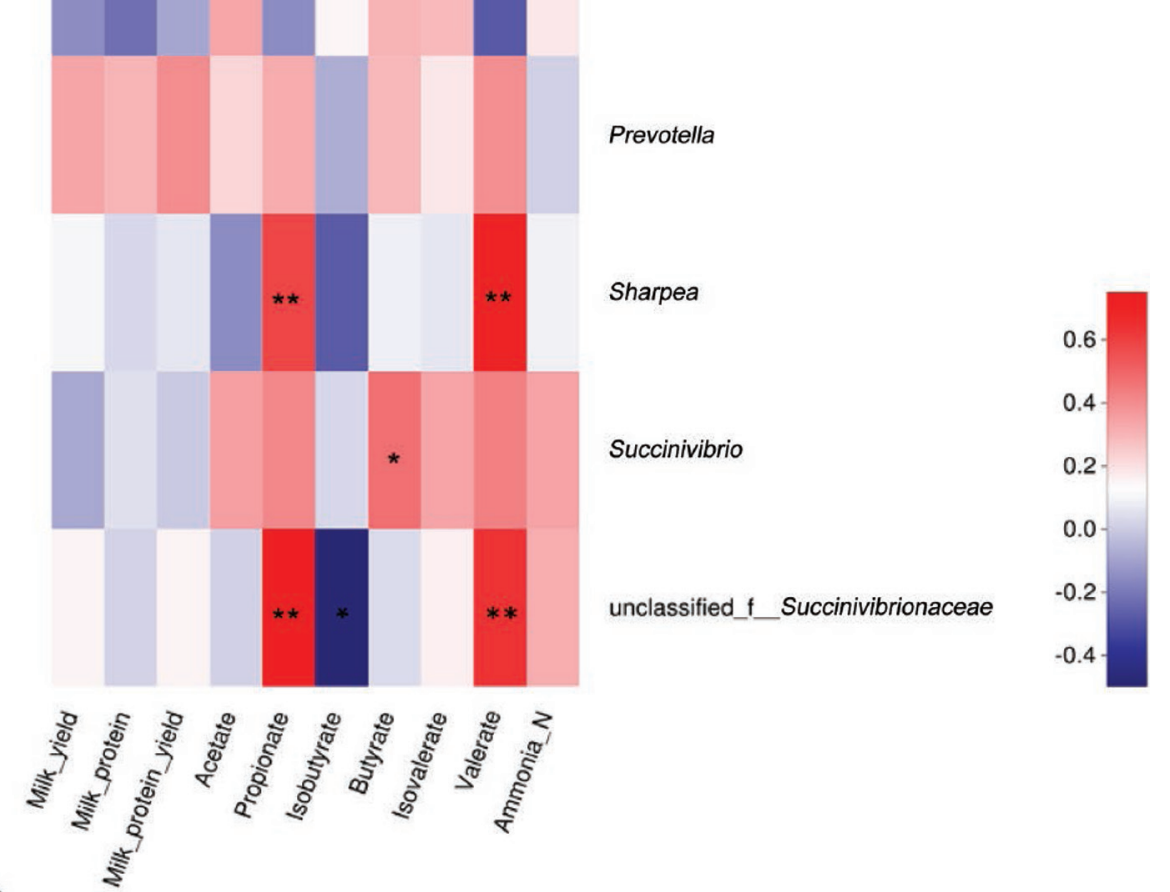

B

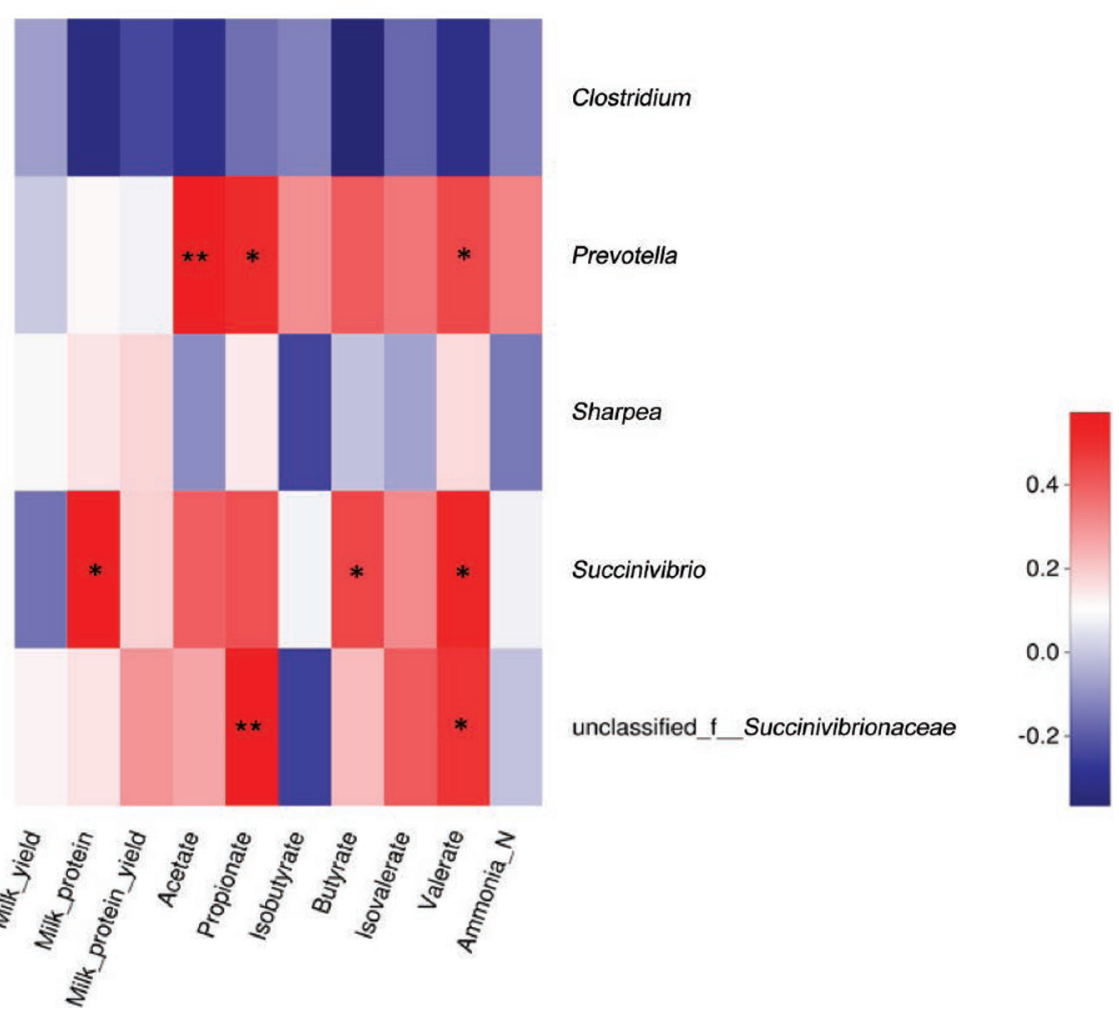

Figure 4. Spearman correlations between specific bacterial genera, VFA, and milking traits. (A) Spearman correlations within dairy cows with high milk yield and high protein yield (HH). (B) Spearman correlations within dairy cows with low milk yield and low protein yield (LL). Significant correlations: ${ }^{*} P<0.05 ; * * P<0.01$. 
al., 2014). Although our results showed that Prevotella contributes to higher VFA concentrations (acetate, propionate, and valerate), our study could not confirm whether members of this genus have roles in MPY. In addition, Clostridium tended to be more abundant in the LL cows than in the HH cows. Previous research showed a negative relationship between Clostridium and milk protein content (Jami et al., 2014), indicating that the abundance of this taxon negatively affects MP and MPY. The members belonging to this genus are cellulolytic, proteolytic, and amylolytic bacteria, which mainly produce acetate and butyrate (Sirohi et al., 2012), but no correlation between Clostridium and VFA concentrations was found in the current study. As the genus Clostridium contains approximately 100 species that may have varied functions, further investigations at deeper taxonomic levels are required to identify the linkages between Clostridium species or strains, VFA concentrations, and MPY.

Surprisingly, the abundance of the genus Sharpea was more than 2.3-fold higher in the HH cows $(0.16 \%)$ than in the LL cows $(0.07 \%)$. Bacteria belonging to this genus were first isolated from the feces of thoroughbred horses (Morita et al., 2008) and have been reported to utilize a wide variety of carbohydrates to produce lactate (Kumar et al., 2018). A recent study has reported the enrichment of lactate-producing Sharpea in rumen bacterial communities of sheep yielding low levels of methane (Kamke et al., 2016). The authors (Kamke et al., 2016) speculated that the higher abundance of Sharpea caused the fermentation shift of converting hexoses to butyrate (mediated by Megasphaera, one of the lactate utilizers, which produce butyrate from lactate; Counotte et al., 1981) in the rumen of animals yielding low levels of methane. In our study, such interactive relationship between Sharpea and Megasphaera was not confirmed in the rumen of the HH cows, as Megasphaera was not detected due to its low relative abundance $(0.0042 \%$ on average, and it existed in only 4 cows). In the meantime, we compared the relative abundances of other lactate-utilizing bacteria, including Anaerovibrio (Mackie and Gilchrist, 1979) and Selenomonas (Gutierrez, 1953), which were not different between the $\mathrm{HH}$ and LL cows. In addition, Propionibacterium (Mackie and Gilchrist, 1979) and Veillonella (Johns, 1951) were not considered as identified due to low relative abundances. Although the relationship between Sharpea and lactate utilizers was not detected in this study, the greater than 2-fold enrichment of Sharpea, the higher propionate and butyrate concentrations in the $\mathrm{HH}$ cows than in the LL cows, and the positive correlations between Sharpea and VFA concentrations (propionate and valerate) indicated that the Sharpea-enriched microbiome in the $\mathrm{HH}$ cows resulted in a fermentation shift toward the production of intermediates (lactate) and end products (butyrate), contributing to high MPY. However, this speculation needs further validation via the characterization of the microbial metabolism and intermediate metabolites in cows with high MPY.

\section{CONCLUSIONS}

Rumen bacterial diversity and abundances of some bacterial taxa significantly differed between dairy cows with high and low MPY. Bacterial richness and relative abundances of Clostridium and Succinivibrio were lower, whereas relative abundances of unclassified Succinivibrionaceae and Sharpea along with VFA concentrations were higher in the rumen of high-MPY cows than in the rumen of low-MPY cows. However, the difference in microbiota composition may not lead to functional changes. Future studies using metagenomic and metatranscriptomic analysis are needed to determine whether the microbial metabolic pathways or metabolites may contribute to MPY. In addition, isolation of the identified microbial taxa and a directly fed microbes-based feeding trial are needed to determine their dominant or causal effects on high MPY.

\section{ACKNOWLEDGMENTS}

The research described herein was supported by grants from the National Natural Science Foundation of China (31729004, Beijing) and the China Agriculture (Dairy) Research System (CARS-36, Beijing). We also acknowledge the members of the Institute of Dairy Science of Zhejiang University (Hangzhou, China) for their assistance in the field sampling and data analysis.

\section{REFERENCES}

Bedö, S., E. Nikodémusz, K. Percsich, and L. Bárdos. 1995. Variations in the milk yield and milk composition of dairy cows during lactation. Acta Vet. Hung. 43:163-171.

Bryant, M. P., and N. Small. 1956. Characteristics of two new genera of anaerobic curved rods isolated from the rumen of cattle. J. Bacteriol. 72:22-26.

Caporaso, J. G., J. Kuczynski, J. Stombaugh, K. Bittinger, and F. Bushman. 2010. QIIME allows integration and analysis of highthroughput community sequencing data. Nat. Methods 7:335-336.

Counotte, G. H. M., R. A. Prins, R. H. A. M. Janssen, and M. J. A. deBie. 1981. Role of Megasphaera elsdenii in the fermentation of DL- $[2-\mathrm{C}]$ lactate in the rumen of dairy cattle. Appl. Environ. Microbiol. 42:649-655.

Duffield, T., J. C. Plaizier, A. Fairfield, R. Bagg, G. Vessie, P. Dick, J. Wilson, J. Aramini, and B. McBride. 2004. Comparison of techniques for measurement of rumen $\mathrm{pH}$ in lactating dairy cows. J. Dairy Sci. 87:59-66.

Edgar, R. C. 2013. UPARSE: Highly accurate OTU sequences from microbial amplicon reads. Nat. Methods 10:996-998.

Edgar, R. C., B. J. Haas, J. C. Clemente, C. Quince, and R. Knight. 2011. UCHIME improves sensitivity and speed of chimera detection. Bioinformatics 27:2194-2200. 
Elolimy, A. A., J. M. Arroyo, F. Batistel, M. A. Iakiviak, and J. J. Loor. 2018. Association of residual feed intake with abundance of ruminal bacteria and biopolymer hydrolyzing enzyme activities during the peripartal period and early lactation in Holstein dairy cows. J. Anim. Sci. Biotechnol. 9:43.

Flint, H. J., and E. A. Bayer. 2008. Plant cell wall breakdown by anaerobic microorganisms from the mammalian digestive tract. Ann. N. Y. Acad. Sci. 1125:280-288.

Geishauser, T., and A. Gitzel. 1996. A comparison of rumen fluid sampled by oro-ruminal probe versus rumen fistula. Small Rumin. Res. 21:63-69.

Gernand, E., and S. Konig. 2014. Short communication: Genetic relationships between claw disorders, protein yield, and somatic cell score by days in milk. J. Dairy Sci. 97:5872-5879.

Gillah, K. A., G. C. Kifaro, and J. Madsen. 2014. Effects of management practices on yield and quality of milk from smallholder dairy units in urban and peri-urban Morogoro, Tanzania. Trop. Anim. Health Prod. 46:1177-1183.

Gutierrez, J. 1953. Numbers and characteristics of lactate utilizing organisms in the rumen of cattle. J. Bacteriol. 66:123-128.

Hernandez-Sanabria, E., L. A. Goonewardene, Z. Wang, O. N. Durunna, S. S. Moore, and L. L. Guan. 2012. Impact of feed efficiency and diet on adaptive variations in the bacterial community in the rumen fluid of cattle. Appl. Environ. Microbiol. 78:1203-1214.

Hu, W. L., J. X. Liu, J. A. Ye, Y. M. Wu, and Y. Q. Guo. 2005. Effect of tea saponin on rumen fermentation in vitro. Anim. Feed Sci. Technol. 120:333-339.

Jami, E., B. A. White, and I. Mizrahi. 2014. Potential role of the bovine rumen microbiome in modulating milk composition and feed efficiency. PLoS One 9:e85423.

Jewell, K. A., C. A. McCormick, C. L. Odt, P. J. Weimer, and G. Suen. 2015. Ruminal bacterial community composition in dairy cows is dynamic over the course of two lactations and correlates with feed efficiency. Appl. Environ. Microbiol. 81:4697-4710.

Johns, A. T. 1951. Isolation of a bacterium, producing propionic acid, from the rumen of sheep. J. Gen. Microbiol. 5:317-325.

Kamke, J., S. Kittelmann, P. Soni, Y. Li, M. Tavendale, S. Ganesh, P. H. Janssen, W. Shi, J. Froula, E. M. Rubin, and G. T. Attwood. 2016. Rumen metagenome and metatranscriptome analyses of low methane yield sheep reveals a Sharpea-enriched microbiome characterized by lactic acid formation and utilization. Microbiome $4: 56$.

Kim, H., I. Lee, Y. Kwon, B. C. Kim, S. Ha, J. H. Lee, and J. Kim. 2011. Immobilization of glucose oxidase into polyaniline nanofiber matrix for biofuel cell applications. Biosens. Bioelectron. 26:39083913.

Krzywinski, M., J. E. Schein, I. Birol, J. Connors, R. Gascoyne, D. Horsman, S. J. Jones, and M. A. Marra. 2009. Circos: An information aesthetic for comparative genomics. Genome Res. 19:16391645 .

Kumar, S., B. P. Treloar, K. H. Teh, C. M. McKenzie, G. Henderson, G. T. Attwood, S. M. Waters, M. L. Patchett, and P. H. Janssen. 2018. Sharpea and Kandleria are lactic acid producing rumen bacteria that do not change their fermentation products when cocultured with a methanogen. Anaerobe 54:31-38.

Li, F., and L. L. Guan. 2017. Metatranscriptomic profiling reveals linkages between the active rumen microbiome and feed efficiency in beef cattle. Appl. Environ. Microbiol. 83:e0061-17.

Lodge-Ivey, S. L., J. Browne-Silva, and M. B. Horvath. 2009. Technical note: Bacterial diversity and fermentation end products in rumen fluid samples collected via oral lavage or rumen cannula. J. Anim. Sci. 87:2333-2337.
Mackie, R. I., and F. M. C. Gilchrist. 1979. Changes in lactate-producing and lactate-utilizing bacteria in relation to $\mathrm{pH}$ in the rumen of sheep during stepwise adaptation to a high-concentrate diet. Appl. Environ. Microbiol. 38:422-430.

Magoč, T., and S. L. Salzberg. 2011. FLASH: Fast length adjustment of short reads to improve genome assemblies. Bioinformatics 27:2957-2963.

Morita, H., C. Shiratori, M. Murakami, H. Takami, H. Toh, Y. Kato, F. Nakajima, M. Takagi, H. Akita, T. Masaoka, and M. Hattori. 2008. Sharpea azabuensis gen. nov., sp. nov., a Gram-positive, strictly anaerobic bacterium isolated from the faeces of thoroughbred horses. Int. J. Syst. Evol. Microbiol. 58:2682-2686.

Nyonyo, T., T. Shinkai, A. Tajima, and M. Mitsumori. 2013. Effect of media composition, including gelling agents, on isolation of previously uncultured rumen bacteria. Lett. Appl. Microbiol. 56:63-70.

Ramos-Morales, E., A. Arco-Pérez, A. I. Martín-García, D. R. YáñezRuiz, P. Frutos, and G. Hervás. 2014. Use of stomach tubing as an alternative to rumen cannulation to study ruminal fermentation and microbiota in sheep and goats. Anim. Feed Sci. Technol. 198:57-66.

Russell, J. B., and J. L. Rychlik. 2001. Factors that alter rumen microbial ecology. Science 292:1119-1122.

Shabat, S. K. B., G. Sasson, A. Doronfaigenboim, T. Durman, S. Yaacoby, M. E. B. Miller, B. A. White, N. Shterzer, and I. Mizrahi. 2016. Specific microbiome-dependent mechanisms underlie the energy harvest efficiency of ruminants. ISME J. 10:2958-2972.

Shen, J. S., Z. Chai, L. J. Song, J. X. Liu, and Y. M. Wu. 2012. Insertion depth of oral stomach tubes may affect the fermentation parameters of ruminal fluid collected in dairy cows. J. Dairy Sci. 95:5978-5984.

Sirohi, S. K., N. Singh, S. S. Dagar, and A. K. Puniya. 2012. Molecular tools for deciphering the microbial community structure and diversity in rumen ecosystem. Appl. Microbiol. Biotechnol. 95:1135-1154

Sova, A. D., S. J. LeBlanc, B. W. McBride, and T. J. DeVries. 2013. Associations between herd-level feeding management practices, feed sorting, and milk production in free stall dairy farms. J. Dairy Sci. 96:4759-4770.

Stevenson, D. M., and P. J. Weimer. 2007. Dominance of Prevotella and low abundance of classical ruminal bacterial species in the bovine rumen revealed by relative quantification real-time PCR. Appl. Microbiol. Biotechnol. 75:165-174.

Terré, M., L. Castells, F. Fabregas, and A. Bach. 2013. Short communication: Comparison of $\mathrm{pH}$, volatile fatty acids, and microbiome of rumen samples from preweaned calves obtained via cannula or stomach tube. J. Dairy Sci. 96:5290-5294.

Wallace, R. J., J. A. Rooke, N. Mckain, C. A. Duthie, J. J. Hyslop, D. W. Ross, A. Waterhouse, M. Watson, and R. Roehe. 2015. The rumen microbial metagenome associated with high methane production in cattle. BMC Genomics 16:839.

Wu, X., H. Z. Sun, M. Y. Xue, D. M. Wang, L. L. Guan, and J. X. Liu. 2018. Serum metabolome profiling revealed potential biomarkers for milk protein yield in dairy cows. J. Proteomics 184:54-61.

Xue, M., H. Z. Sun, X. H. Wu, L. L. Guan, and J. X. Liu. 2018. Assessment of rumen microbiota from a large cattle cohort reveals the pan and core bacteriome contributing to varied phenotypes. Appl. Environ. Microbiol. 84:e00970-18.

$\mathrm{Yu}, \mathrm{Z}$., and M. Morrison. 2004. Improved extraction of PCR-quality community DNA from digesta and fecal samples. Biotechniques $36: 808-812$. 